\title{
Impact of Fiber Structure on the Material Stability and Rupture Mechanisms of Coronary Atherosclerotic Plaques
}

\author{
Graeham R. Douglas, ${ }^{1}$ Adam J. Brown, ${ }^{2}$ Jonathan H. Gillard, ${ }^{3}$ Martin R. Bennett, ${ }^{2}$ \\ Michael P. F. Sutcliffe, ${ }^{1}$ and Zhongzhao Teng (i) ${ }^{1,3}$ \\ ${ }^{1}$ Department of Engineering, University of Cambridge, Trumpington Street, Cambridge CB2 1PZ, UK; ${ }^{2}$ Division of \\ Cardiovascular Medicine, University of Cambridge, Cambridge, UK; and ${ }^{3}$ Department of Radiology, School of Clinical \\ Medicine, University of Cambridge, Box 218, Cambridge Biomedical Campus, Cambridge CB2 0QQ, UK
}

(Received 24 November 2016; accepted 22 March 2017; published online 30 March 2017)

Associate Editor Andreas Anayiotos oversaw the review of this article.

\begin{abstract}
The rupture of an atherosclerotic plaque in the coronary circulation remains the main cause of heart attack. As a fiber-oriented structure, the fiber structure, in particular in the fibrous cap (FC), may affect both loading and material strength in the plaque. However, the role of fiber orientation and dispersion in plaque rupture is unclear. Local orientation and dispersion of fibers were calculated for the shoulder regions, mid FC, and regions with intimal thickening (IT) from histological images of 16 human coronary atherosclerotic lesions. Finite element analysis was performed to assess the effect of these properties on mechanical conditions. Fibers in shoulder regions had markedly reduced alignment (Median [interquartile range] $12.9^{\circ}[6.6,18.0], p<0.05$ ) compared with those in mid FC $\left(6.1^{\circ}[5.5,9.0]\right)$ and IT regions $\left(6.7^{\circ}[5.1,8.6]\right)$. Fiber dispersion was highest in shoulders $(0.150[0.121,0.192])$, intermediate in IT $(0.119$ $[0.103,0.144])$, and lowest in mid FC regions $(0.093$ [0.081, $0.105], p<0.05)$. When anisotropic properties were considered, stresses were significantly higher for the mid FC $(p=0.030)$ and IT regions $(p=0.002)$ and no difference was found for the shoulder or global regions. Shear (sliding) stress between fibers in each region and their proportion of maximum principal stress were: shoulder $(25.8 \mathrm{kPa}$ [17.1, 41.2], 12.4\%), mid FC (13.9 $\mathrm{kPa}[5.8,29.6], 13.8 \%)$, and IT $(36.5 \mathrm{kPa}[25.9,47.3], 15.5 \%)$. Fiber structure within the FC has a marked effect on principal stresses, resulting in considerable shear stress between fibers. Fiber structure including orientation and dispersion may determine mechanical strength and thus rupture of atherosclerotic plaques.
\end{abstract}

Keywords-Coronary, Atherosclerosis, Rupture, Fiber, Stress.

Address correspondence to Michael P. F. Sutcliffe, Department of Engineering, University of Cambridge, Trumpington Street, Cambridge CB2 1PZ, UK and Zhongzhao Teng, Department of Radiology, School of Clinical Medicine, University of Cambridge, Box 218, Cambridge Biomedical Campus, Cambridge CB2 0QQ, UK. Electronic mails: mpfs@eng.cam.ac.uk, zt215@cam.ac.uk

\section{INTRODUCTION}

Heart disease remains the first global cause of death and the number is expected to rise to more than 23.6 million each year by $2030 .{ }^{26}$ Deaths from heart disease are mainly due to coronary atherosclerotic disease. ${ }^{49} \mathrm{Au}$ topsy studies demonstrated that many myocardial infarctions were caused by rupture of a coronary atherosclerotic plaque exhibiting a large lipid-rich necrotic core and luminal thrombus. ${ }^{48}$ The proposed precursor lesion for rupture displays a similar architecture of a thin fibrous cap (FC) separating the lipid core from the lumen, termed a thin-cap fibroatheroma (TCFA). ${ }^{6}$ Clinical and angiographic imaging, including computerized tomography and digital subtract angiography, cannot identify TCFA or other higher risk plaques, ${ }^{3}$ indicating a major need for diagnostic modalities that can identify and stratify higher-risk plaques prior to rupture.

Prospective studies utilizing virtual histology intravascular ultrasound (VH-IVUS)-defined plaque classification algorithms, including PROSPECT (A prospective natural-history study of coronary atherosclerosis), Virtual Histology in Vulnerable Atherosclerosis (VIVA), and ATHEROREMO-IVUS, have shown an association between TCFA and subsequent major adverse cardiovascular events. ${ }^{7,10,37}$ Although these studies suggest that VH-IVUS can prospectively identify higher-risk plaques, the absolute event rate per lesion identified as higher-risk was low $(<10 \%$ at 3 years). Thus, additional methods for assessment of plaque vulnerability are required to improve our ability to predict plaque rupture including higher risk features captured by other imaging modalities, e.g., optical coherence tomography. ${ }^{36,50}$ 
Under physiological conditions, atherosclerotic lesion is subject to mechanical loading induced by blood pressure $^{38,42}$ and flow. Experimental and computational studies have shed light to the importance of endothelial shear stress in progressive atherosclerosis and rupture. Some of these studies have been summarized elsewhere. ${ }^{46}$ On the other hand, high structural stress concentration within the plaque, which is about $10^{3}-10^{5}$ times higher than endothelial shear stress, has been shown to be associated with fissuring and rupture in both coronary ${ }^{29}$ and carotid ${ }^{38}$ plaques. Structural stress and stretch may also differentiate patient clinical presentations, ${ }^{15,41,53}$ and are associated with subsequent cerebrovascular $^{32,44}$ and heart ischemic events in symptomatic patients. ${ }^{5}$ In general, stable lesions are protected by a thick FC, as increasing FC thickness results in decreasing structural stress. ${ }^{25,52}$ Autopsy studies have shown that around $60 \%$ of $\mathrm{FC}$ ruptures are located in the plaque shoulder region, where luminal curvature is sharper. ${ }^{29}$ Failure at the shoulder can be partially explained by pronounced luminal angulation leading to an increased stress level in the FC. ${ }^{43}$ However, as an anisotropic material with collagen and elastin fibers the primary mechanical components, ${ }^{8,20,34}$ the fine structures within the FC may be an important determinant in FC stability. ${ }^{39}$ Advancing states of atherosclerosis are known to alter the structure, organization, and concentration of collagen fibers within the tissue matrix. ${ }^{34}$ However, the impact from changes in fiber structure on critical mechanical conditions within atherosclerosis remains unexplored.

Within the cross-section of a healthy artery, fibers are concentric and have a primary orientation aligned with the local lumen tangent. ${ }^{17}$ Atherosclerotic progression often results in an eccentric, non-circular lumen along with disruption to fiber patterns from lipid deposits and intraplaque hemorrhage. In this study, orientation of predominately collagen fibers was extracted from histological images of coronary atherosclerotic plaques. Fiber misalignment was calculated as the local orientation relative to the luminal tangent. The local distribution of fiber orientation relative to their primary orientation was used to calculate fiber dispersion. Fiber misalignment and dispersion were compared between anatomical plaque regions. Two-dimensional finite element (FE) models were reconstructed using the geometry and fiber orientation from the histological images, with loading from an assumed static internal blood pressure. To suggest novel mechanisms for FC failure, mechanical stress was decomposed into components along (axial), perpendicular to (transverse), and shearing between the fibers. The relationship between critical mechanical conditions and fiber architecture was explored.

\section{MATERIALS AND METHODS}

\section{Specimen and Histological Processing}

Arteries with atherosclerotic plaques were harvested from human hearts during autopsy in consultation with a senior pathologist. The study protocol was approved by the Cambridgeshire Research and Ethics Committee (Ref. 07/H0306/123) and consent obtained from relatives. Sixteen left anterior descending arteries were dissected, excised, sectioned, and stained with hematoxylin and eosin (H\&E) and Verhoeff's Van Gieson (EVG). More details about the specimens and histological processing can be found in our previous report. ${ }^{4}$ Histology slides were digitized using Nanozoomer (Hamamatsu Photonics KK, Hamamatsu, Japan) under $40 \times$ magnification.

\section{Image Processing}

Histological slides from the proximal section of each plaque, where most ruptures occur, were carefully reviewed, and the best slide, without serious artefacts and damage induced by histological processing, was included in the analysis. Image segmentation was performed by an experienced cardiac pathologist to identify atherosclerotic plaque components, including FC and lipid (Fig. 1a). The region adjacent to the lumen was divided into sub-regions of shoulder, mid FC, and intima thickening (IT) to perform location-dependent analyses (Fig. 1a). In this study, unless otherwise specified, FC included fibrous tissues in the shoulder region and mid FC.

Each image was exported from NDP.view 2.3.0 (Hamamatsu Photonics KK, Hamamatsu, Japan) in bitmap format with pixel size of $3.5 \times 3.5 \mu \mathrm{m}^{2}$ in preparation for further processing to quantify the fiber orientation and dispersion (Figs. 1 and 2). Fibers were assumed to be slender structures with strong contrast against their background and were identified as edges in the image. Details of methods used to identify and to calculate the orientation and dispersion (i.e. the spread) of fibers are included in the Supplemental Material.

\section{Finite Element Analysis}

Segmented contours of the lumen, lipid region, and outer edge of the plaque were used to construct twodimensional (2D) FE models for each image. Two comparative FE models were built. First, an isotropic analysis assumed that fibers were evenly scattered in all directions. Second an anisotropic analysis assigned fiber orientation by element. 

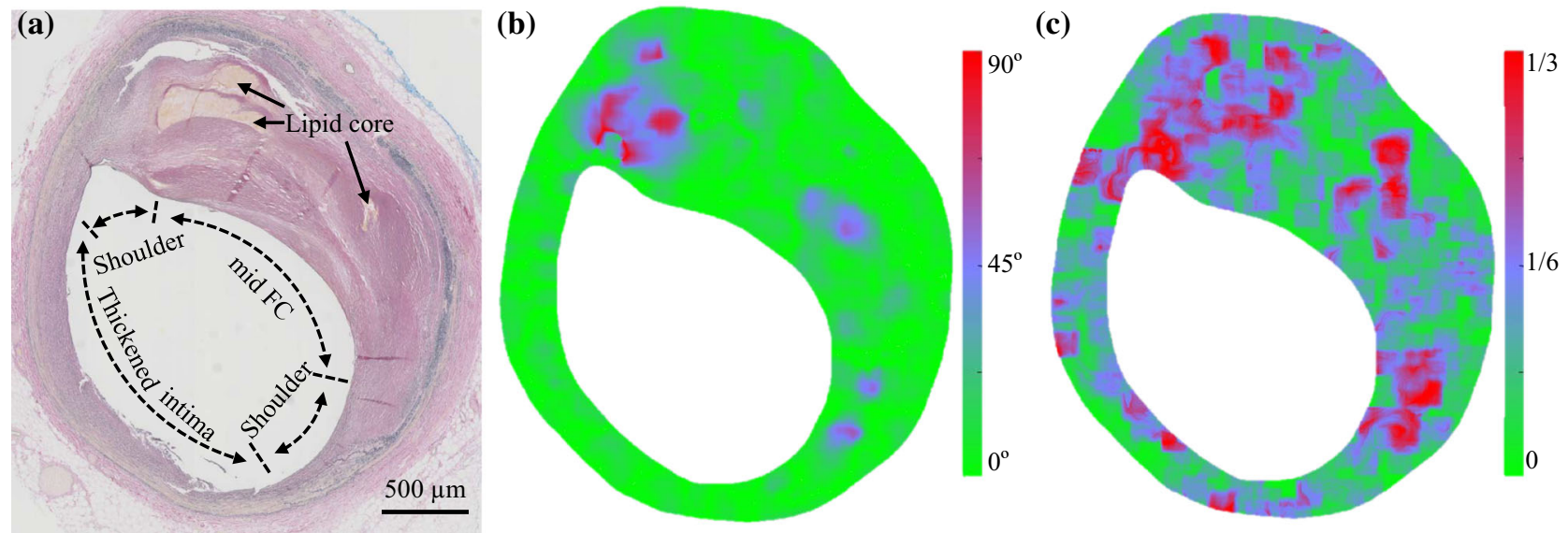

FIGURE 1. Representative histology image and corresponding distribution of fiber orientation and dispersion ((a) EVG-stained image labelled by tissue regions [Shoulder, mid FC, and region with intima thickening (IT)]; (b) Map of fiber orientation [greener color: better alignment with lumen]; and (c) Map of fiber dispersion [greener color: less fiber dispersion]).

(a) $\kappa=0.330$

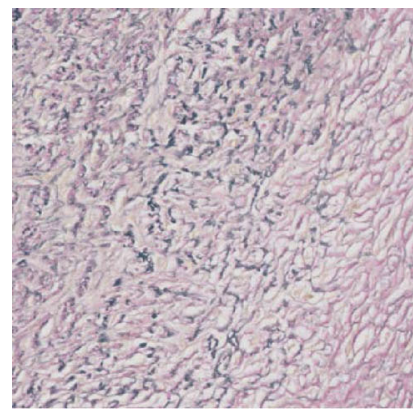

(c) $\kappa=0.136$

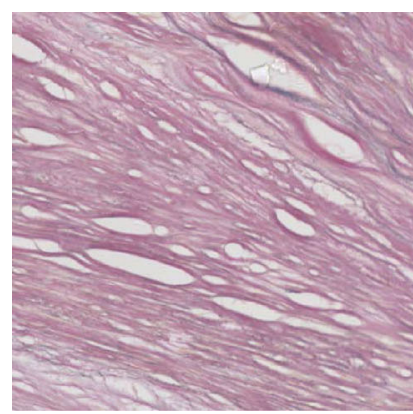

(b) $\kappa=0.234$
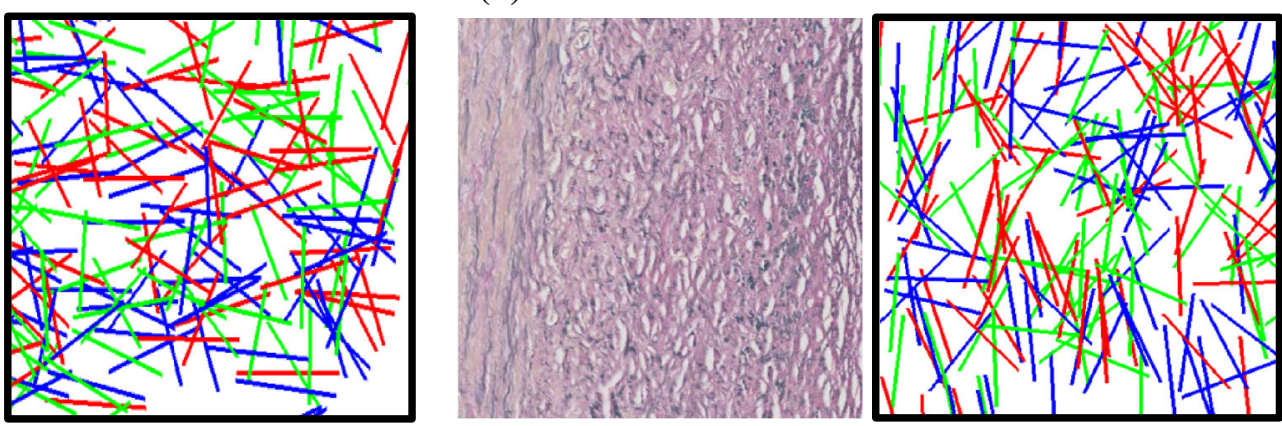

(d) $\kappa=0.054$

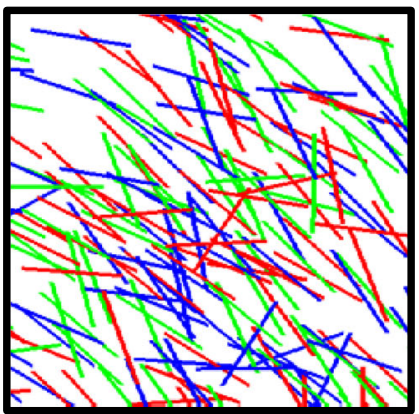

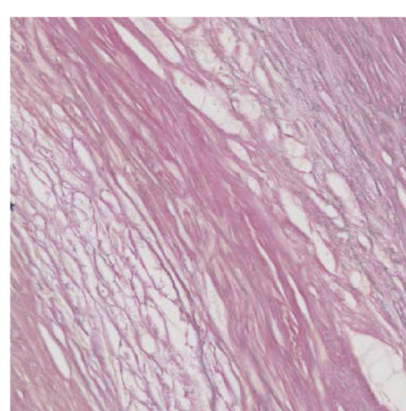

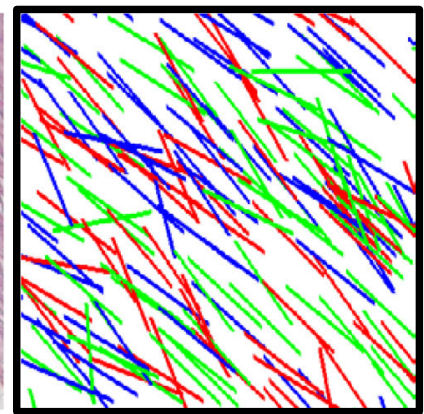

FIGURE 2. Histology enlargement and generated plot of synthetic fibers with the same orientation and dispersion ((a) a region with nearly even distribution of fiber orientation; (b) a region with less evenly distributed fiber orientation; (c) a region with relatively less spread in fiber orientation; and (d) a region with very little spread in fiber orientation; $\kappa \in[0,1 / 3]$ indicates the extent of fiber dispersion, larger $\boldsymbol{\kappa}$ for more misalignment of fibers).

The fibrous tissue was modeled as a Gasser-OgdenHolzapfel anisotropic, incompressible hyperelastic solid, ${ }^{17}$ defined by the strain energy density function, $\psi$ :

$\psi=C_{1}\left(I_{1}-3\right)+\frac{k_{1}}{2 k_{2}}\left\{\exp \left[k_{2}\left(\kappa I_{1}+(1-3 \kappa) I_{4}-1\right)^{2}\right]-1\right\}$ in which $I_{1}$ is the first invariant of the deformation tensor. The fiber structure is described by invariant $I_{4}=\left(\lambda_{1} \cos \alpha\right)^{2}+\left(\lambda_{2} \sin \alpha\right)^{2}$, where $\alpha$ is the fiber orientation within the cross-sectional plane of the FE model, and $\lambda_{1}$ and $\lambda_{2}$ are the stretch ratios along and perpendicular to the local fiber orientation, respectively. Since fiber orientation was assigned by element, 
fiber dispersion was accounted for by the local value of $\alpha$ as well as a material fiber dispersion of $\kappa=0.136$. The remaining parameters are material constants that used values from a previous study for carotid plaque tissue $^{19}$ : the linear isotropic stiffness of the ground matrix is $C_{1}=0.056 \mathrm{MPa}$; the strain-stiffening response of the fibers is described by $k_{1}=41.08 \mathrm{MPa}$ and $k_{2}=1749.6$.

The lipid tissue was modeled as an isotropic, incompressible hyperelastic solid with a reduced polynomial strain energy density function, $\phi$ :

$$
\phi=A_{1}\left(I_{1}-3\right)+A_{2}\left(I_{1}-3\right)^{2}
$$

in which $A_{1}=0.0225 \mathrm{MPa}$ and $A_{2}=0.2150 \mathrm{MPa}$, obtained through fitting uniaxial-extension stressstretch curves from lipid tissues strips from human carotid atheromas. ${ }^{45}$

The geometry was meshed using edge seeds of $5.0 \mu \mathrm{m}$ on the lumen, $10.0 \mu \mathrm{m}$ on the lipid edge, and $50.0 \mu \mathrm{m}$ on the outer edge using the Abaqus FE package (Abaqus 6.13-2, Dassault Systèmes, France). The leftmost point of the outer wall was fixed to prevent rigid body movements and an internal pressure of $16 \mathrm{kPa}(120 \mathrm{mmHg})$ was applied to the lumen surface. Unrealistically high stress might be calculated in FE models from sharp geometries or fiber discontinuities. Therefore, reported stress for each segmented region was the stress that at least $2500 \mu \mathrm{m}^{2}$ of the region surpassed. This filter ignored about 15-30 higheststress elements in each region. Isotropic maximum principle stress (Stress- $\mathrm{P}_{1}$ ) from the isotropic model, and anisotropic Stress- $\mathrm{P}_{1}$, axial, transverse, and shear stress from the anisotropic model were extracted for analysis. A schematic drawing for each stress component is shown in Fig. 3.

\section{Statistical Analysis}

A paired $t$ test or Wilcoxon-Mann-Whitney test was used where appropriate to assess the difference in fiber orientation, dispersion, and critical mechanical conditions between different plaque regions. A significant difference was assumed if $p<0.05$.

Simple linear regressions explored the relationship between fiber characteristics (fiber misalignment and dispersion), local geometrical factors, and finite element results, including isotropic Stress- $\mathrm{P}_{1}$, anisotropic Stress- $\mathrm{P}_{1}$, axial, transverse, and shear stress, for regions around the lumen. These models report the unit-dependent slope of the regression; the p-value for this slope being zero (or no statistical relationship); and a regression coefficient $\left(\mathrm{R}^{2}\right)$ that describes how much of the output variability is predicted by the independent parameter. As there are complex interde- pendencies between geometry, fiber structure, and mechanics, the regression coefficient can be small for significant relationships $(p<0.05)$ between variables. Geometry parameters considered in this study are defined in Table 1.

Multivariate regressions analyzed these complex interdependencies to better predict anisotropic stresses. From an initial regression of isotropic Stress- $P_{1}$ against an anisotropic stresses, geometry and fiber parameters were added to the regression if doing so increased the $\mathrm{R}^{2}$ by at least 0.1 . All statistical and regression analyses were performed in Matlab R2016a (The MathWorks, Inc., USA).

\section{RESULTS}

\section{Fiber Orientation and Dispersion}

As shown in the illustrative plaque (Fig. 1a), fibers in the narrow region adjacent to the lumen boundary were predominately oriented parallel to the boundary (Fig. 1b), although the orientation deeper in the plaque is less aligned with the lumen. In contrast, fibers in the shoulder region were not well aligned and in some cases were perpendicular to the local lumen edge (Fig. 1b). Quantification of orientation showed better alignment of fibers with the lumen boundary in the mid FC and IT than the shoulder region (Table 2). Dispersion was highest in the shoulders, intermediate in the IT region, and lowest in the mid FC (Fig. 1c and Table 2). For reference, a fiber dispersion of $\kappa=1 / 3$ is an evenly scattered set of fiber orientations (the local material is isotropic) and $\kappa=0$ indicates that all fibers are parallel. Figure 2 shows a series of magnified histological sections across a range of fiber dispersions, paired with plots of synthetic fiber having the same fiber orientation and dispersion.

\section{Differences in Stress- $P_{1}$ Between Isotropic and Anisotropic Models}

Representative maximum principal stress (Stress- $\mathrm{P}_{1}$ ) plots from isotropic and anisotropic models are shown in Fig. 4a and 4b. Stresses were usually higher near the lumen in both models. In the isotropic models, the stresses were often highest and concentrated in the shoulder regions, where luminal curvature was tighter. In the anisotropic models, higher stress zones formed slender bands, which extended deeper into the tissue towards and diverged around the lipid core (Fig. 4b; Supplemental Fig. S1). We examined global and regional stress using both isotropic and anisotropic models. There was no significant difference between 

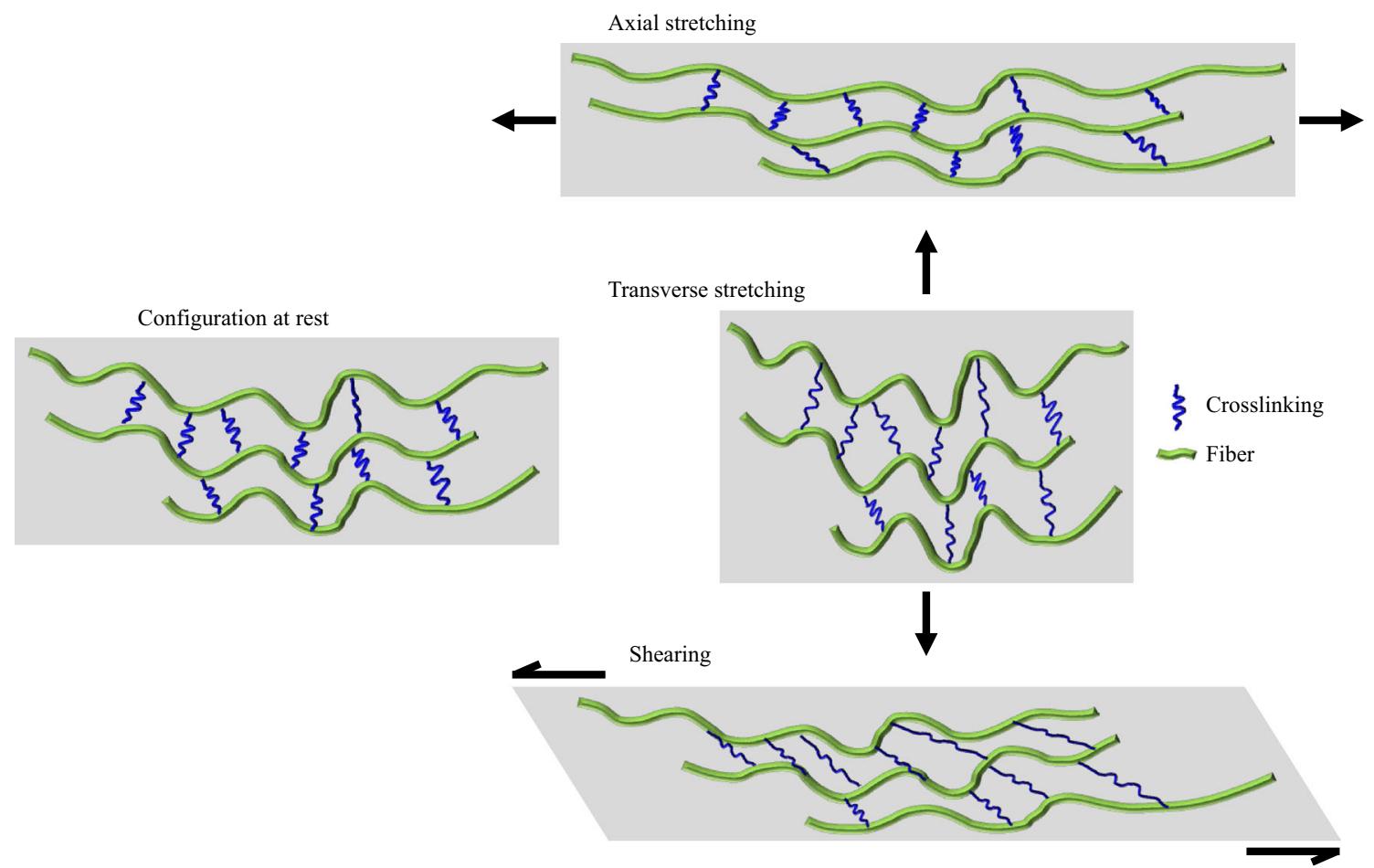

FIGURE 3. Schematic drawing of a fibrous material under different loading ((a) at rest; (b) stress along the fibers (axial stress); (c) stress perpendicular to the fibers (transverse stress); and (d) stress shearing the fibers (shear stress)).

TABLE 1. Definition of geometry properties used in linear modelling.

Parameter name

Plaque burden (\%)

Plaque area $\left(\mathrm{mm}^{2}\right)$ Lumen area $\left(\mathrm{mm}^{2}\right)$

Lipid area $\left(\mathrm{mm}^{2}\right)$

Lipid fraction (\%)

Lipid thickness $(\mathrm{mm})$

Lipid arc $\left(^{\circ}\right)$

FC thickness $(\mathrm{mm})$

Shoulder curvature $\left(\mathrm{mm}^{-1}\right)$

Normalized shoulder curvature
Proportion of plaque area to total area within the external elastic lamella (EEL: boundary between media and adventitia)

Area of tissue within the EEL excluding the lumen

Area within the artery wall

Area of the manually segmented lipid-rich core

Proportion of lipid area to total area with the EEL

Maximum radial thickness of the lipid-rich core

Maximum circumferential arc of the lipid-rich core

Minimum radial thickness of the fibrous cap

Inverse of the median radius of lumen curvature at the shoulder regions. A higher curvature indicates sharper geometry. (For linear regressions within the shoulder region, the same shoulder was used for curvature as for the fiber or FE result being modelled. For regressions within the mid FC and IT, the lower of the two shoulders' curvature was used)

The shoulder curvature, normalized by the diameter of the EEL in the vertical direction of the plaque image

TABLE 2. Fiber orientation and dispersion in the regions along the lumen (data presented as median [interquartile range]).

\begin{tabular}{lccc}
\hline & Shoulder & Mid FC & IT \\
\hline Fiber orientation & $12.9^{\circ}[6.6,18.0]^{*}$ & $6.1^{\circ}[5.5,9.0]$ & $6.7^{\circ}[5.1,8.6]$ \\
Fiber dispersion & $0.150[0.121,0.192]$ & $0.093[0.081,0.105]^{\dagger}$ & $0.119[0.103,0.144]^{\star}$ \\
\hline
\end{tabular}

*Fibers in the shoulder region had more misalignment than those either in the mid FC or IT $(p<0.05)$.

${ }^{\dagger}$ Fibers in the mid FC had less dispersion than those in either the shoulders or IT $(p<0.05)$.

${ }^{*}$ Fibers in the IT region had less dispersion than those in the shoulders $(p<0.05)$. 
the isotropic and anisotropic models for global Stress$\mathrm{P}_{1}$ (Fig. 5 and Table 3). In sub-regions along the lumen, significant differences in Stress- $\mathrm{P}_{1}$ between the two models were found for the IT regions and in the mid FC, but not for the shoulder regions. Stress- $\mathrm{P}_{1}$ was higher for the shoulder compared to the mid FC regions in 15/16 of the isotropic models and 14/16 of the anisotropic models.

\section{Axial, Transverse, and Shear Stress Derived from the Anisotropic Model}

Anisotropic models had components of stress along fibers (axial stress), across fibers (transverse stress), and sliding between fibers (shear stress) (Fig. 4 and Table 4). For clarity, the deformation of fibers and their crosslinks produced by these stresses is illustrated by a cartoon (Fig. 3). Plaques mainly undertake mechanical loading along consecutive fibers, as indicated by axial stress being the largest component (Fig. 4 and Table 4). Higher axial stress was found in the shoulder and IT regions than in the mid FC. Both transverse and shear stresses in each sub-region was significantly lower than the axial stresses (Fig. 6 and Table 4). Transverse stresses in the mid FC, shoulder, and IT regions were all significantly lower than their respective shear stresses. Transverse stress was mostly negative (compressive) around the lumen. Considerable shear stress existed in the regions around the lumen as a proportion of the respective region's peak Stress- $\mathrm{P}_{1}$ : mid FC $(13.8 \%$ [0.10\%, 27.4\%]), shoulder $(12.4 \%[8.8 \%, 23.2 \%])$, and IT $(15.5 \% \quad[10.1 \%$, $19.6 \%]$ ). Anisotropic stress components were higher in the shoulder regions than the mid FC for most samples: axial-14/16, transverse-16/16 including one tied, and shear-14/16.

\section{Geometry Parameters and Fiber Structure}

Only regressions finding significant associations between geometry parameters and fiber structures are reported in Table 5. Fiber misalignment in the shoulder regions was significantly linked to increases in the normalized shoulder curvature, plaque burden, and FC thickness. Fiber dispersion in the shoulders was predicted by increased normalized shoulder curvature and decreased FC thickness. Higher FC thickness was the only predictor of fiber misalignment in the mid FC, and lower lipid fraction the only link with dispersion. No significant links between fiber structure and geometry parameters were found for the IT region.

\section{Geometry Parameters and Finite Element Analysis Results}

For the shoulder region, regressions linked increasing isotropic Stress- $\mathrm{P}_{1}$ to increasing shoulder curvature and normalized shoulder curvature (Table 6). Transverse stresses increased with shoulder curvature and normalized shoulder curvature. No significant links were found between geometry and anisotropic Stress- $\mathrm{P}_{1}$, axial stress, or shear stresses in the shoulder region. For the mid FC, regressions found stresses of all reported types decreased with shoulder curvature and normalized shoulder curvature. For all stress types except shear, stresses increased for wider lipid arcs. Lipid arc is similar to the length of the FC. Shear stress increased with lipid area and lumen area. No significant links between geometry parameters and FE results were found for the IT region.

\section{Fiber Structure and Finite Element Analysis Results}

Simple linear regressions only found one statistically significant coupling between fiber structures (misalignment and dispersion) and FE results. Increased dispersion was a predictor of transverse stress in the shoulders (slope $=153.3, \quad \mathrm{R}^{2}=0.216$, $p=0.007$ ). No other fiber structure parameter was significantly linked to FE results.

\section{Isotropic Principle Stress and Other Stresses}

As the isotropic FE models are a function of geometry and material properties only (i.e. does not have fiber structure), isotropic Stress- $\mathrm{P}_{1}$ was also considered as a variable for linear regressions against the anisotropic FE. Thus, results of the regression imply the degree to which fiber structure affects anisotropic stresses. Regressions indicated that isotropic Stress- $\mathrm{P}_{1}$ was a significant predictor for anisotropic stresses in all regions, except shear and transverse stress in the IT region (Table 7). Isotropic Stress- $\mathrm{P}_{1}$ alone explains the majority of anisotropic Stress- $\mathrm{P}_{1}$ and axial stress variability in the mid FC and IT regions $\left(\mathrm{R}^{2}=0.695-0.712\right)$.

Other anisotropic stresses, especially those in the shoulder region, had only weak or moderate correlations with isotropic Stress- $\mathrm{P}_{1}$ (low $\mathrm{R}^{2}$ ), suggesting fiber architecture caused the considerable changes to the anisotropic FE results. As fiber misalignment and dispersion were not strongly linked to FE results as individual parameters, the role of fiber structure in mechanics appears to be complex. 
(a) Stress- $\mathrm{P}_{1}$ (isotropic model)

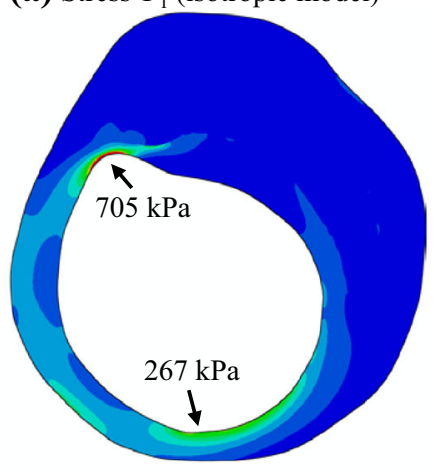

(d) Transverse stress

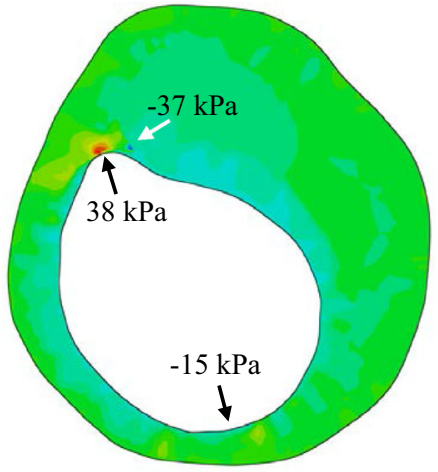

(b) Stress- $\mathrm{P}_{1}$ (anisotropic model)

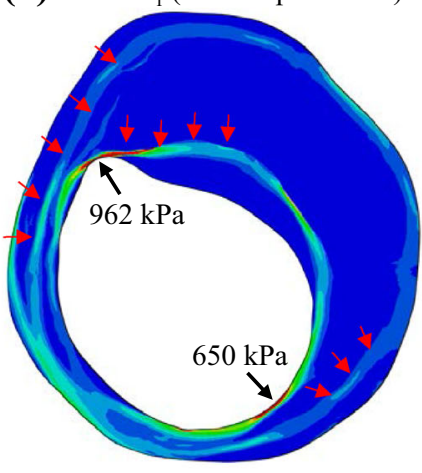

(e) Shear stress

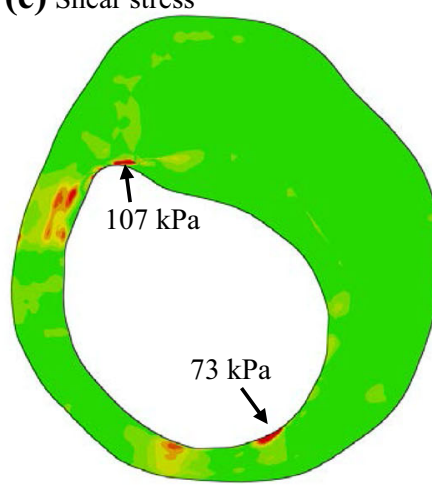

(c) Axial stress
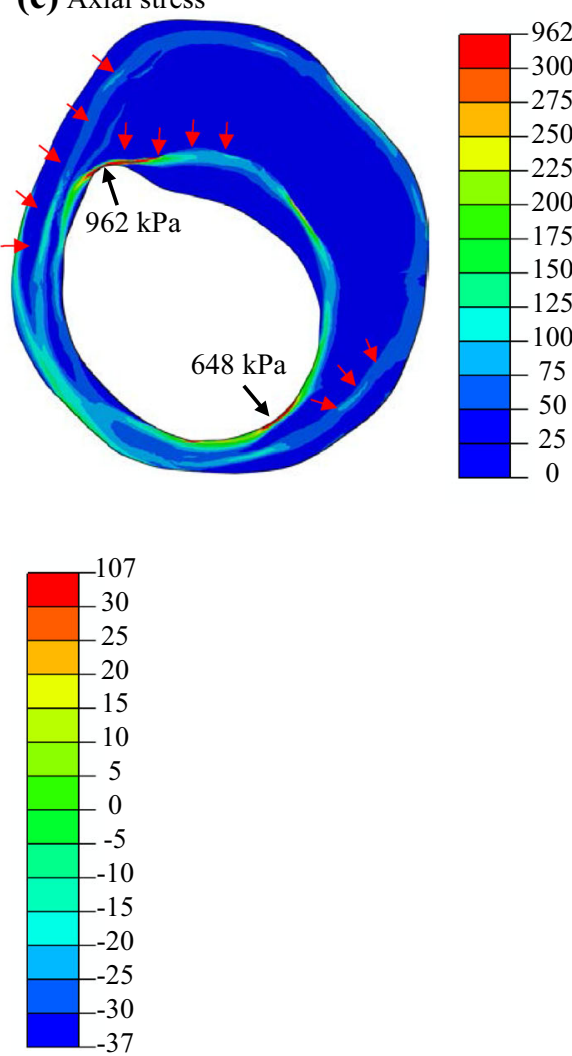

FIGURE 4. Band plot of stress under lumen pressure ((a) Stress- $P_{1}$ predicted using the isotropic model; (b) Stress- $P_{1}$ predicted using the anisotropic model; (c) Stress along the fibers [axial stress]; (d) Stress perpendicular to the fibers [transverse stress]; and (e) Stress shearing the fibers [shear stress]; red arrows in B and C identify slender bands of high stress).

\section{Multivariate Regressions for Anisotropic Stresses}

The multivariate regressions identified potential improvements to linear regression models of anisotropic stresses against isotropic Stress- $\mathrm{P}_{1}$ alone. In the mid FC, a multivariate regression using isotropic Stress- $\mathrm{P}_{1}$ and lipid area improved the regressions against transverse ( $\mathrm{R}^{2}$ increased from 0.670 to 0.828$)$ and shear stresses $\left(\mathrm{R}^{2}\right.$ increased from 0.432 to 0.700 ). For the shoulders, a multivariate regression including FC thickness in addition to isotropic Stress- $\mathrm{P}_{1}$ improved regressions for anisotropic Stress- $\mathrm{P}_{1}\left(\mathrm{R}^{2}\right.$ increased from 0.316 to 0.424$)$ and axial stress ( $\mathrm{R}^{2}$ increased from 0.301 to 0.410$)$. No other multivariate regressions for anisotropic stresses increased $\mathrm{R}^{2}$ by at least 0.1 compared to a linear regression against isotropic Stress- $\mathrm{P}_{1}$ only.

\section{DISCUSSION}

This study sought to quantify fiber orientation and dispersion in coronary atherosclerotic plaques and the impact of these fiber structures on the critical mechanical conditions when pressurized. We showed that fibers were more organized and more aligned with the local lumen boundary in the mid FC and IT, and less organized and less aligned in the plaque shoulder region (Table 2). In addition to the effect of local luminal curvature, such differences in fiber structure may contribute to the high stresses in the shoulder region where most $\mathrm{FC}$ ruptures $(\sim 60 \%)$ occur. ${ }^{29}$ In this study, high stress appearing in an area at least $2500 \mu \mathrm{m}^{2}$ was extracted for analysis. This threshold acted as a filter to avoid reporting very high elemental stress, which might be due to mesh distortion or local imperfect geometric configuration, e.g. sharp curvature. The criteria ignored about $15-30$ of the higheststress elements in each plaque region being equivalent to around the 95-99 percentile of stress in a plaque region. We also have calculated the 95 percentile stress and obtained the same conclusions.

Fiber groups often bifurcate around the lipid core in the shoulder region (Fig. 1a and 1b). Tangency is usually maintained with the lumen boundary and the lipid core, but the fibers are clearly not concentric in the 2D-plane as they are in healthy arteries. Seam-like features traverse from the lumen near the shoulders towards the ends of the lipid core, defining the border between fiber groups diverging to either side of the 

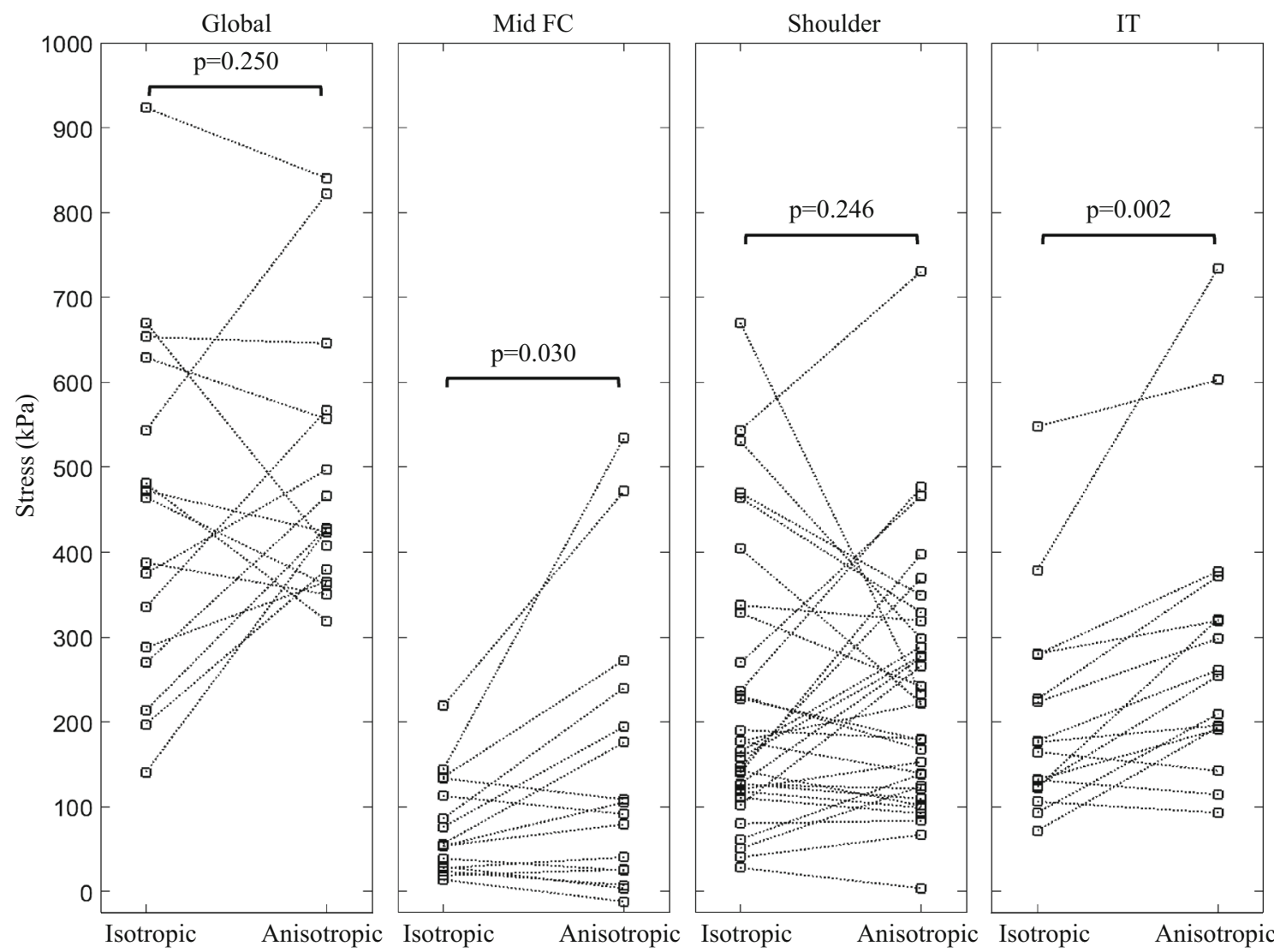

FIGURE 5. Comparison of Stress- $P_{1}$ between the isotropic and anisotropic models, compared globally and in different plaque regions (Global peak stress and the stress value in the shoulder region calculated by both models were comparable; the stress values in mid FC and IT were underestimated by the isotropic model).

TABLE 3. Region-by-region comparison of maximum principal stress (Stress- $P_{1}$ ) between the isotropic and anisotropic models (median, [interquartile range]; unit: $\mathbf{k P a}$ ).

\begin{tabular}{lccr}
\hline & Isotropic Stress- $\mathrm{P}_{1}$ & Anisotropic Stress- $\mathrm{P}_{1}$ & $p$ value \\
\hline Global peak stress & $426.3[278.8,586.4]$ & $427.8[372.5,562.0]$ & 0.250 \\
Mid FC & $55.1[28.6,123.2]$ & $98.4[25.6,217.2]$ & 0.030 \\
Shoulder & $161.4[119.5,299.2]$ & $221.9[122.9,308.6]$ & 0.246 \\
IT & $170.4[123.8,253.2]$ & $257.9[192.6,346.8]$ & 0.002 \\
\hline
\end{tabular}

TABLE 4. Region-by-region comparison of components of anisotropic stress (median, [interquartile range]; unit: kPa).

\begin{tabular}{lccc}
\hline & \multicolumn{1}{c}{ Axial stress $^{\dagger}$} & Transverse stress & Shear stress $^{\star}$ \\
\hline Mid FC & $94.2[24.6,213.4]$ & $-3.9[-11.3,4.0]$ & $13.9[5.8,29.6]$ \\
Shoulder & $212.2[122.3,302.3]^{\star}$ & $9.6[0.3,15.2]$ & $25.8[17.1,41.2]$ \\
IT & $256.9[187.9,332.0]^{*}$ & $9.8[2.9,13.7]$ & $36.5[25.9,47.3]$ \\
\hline
\end{tabular}

${ }^{\dagger}$ Axial stresses for a respective region were significantly higher than transverse or shear stresses for the same region $(p<0.0001)$.

\$Shear stresses for a respective region were significantly higher than transverse stresses for the same region $(p<0.0001)$.

${ }^{*}$ Axial stresses in the shoulder and IT were significantly higher than in the mid FC $(p<0.05)$.

lipid core. These diverging fibers are highly misaligned and dispersed, as in the left shoulder of Fig. 1b. This may explain why fibers in the shoulder region had higher misalignment and dispersion. The seams follow a similar trajectory as the slender bands of high stress between the shoulder and lipid core, noted in the anisotropic model (Fig. 4b and 4c). The bands have highest stress at the lumen border of the shoulder, but 


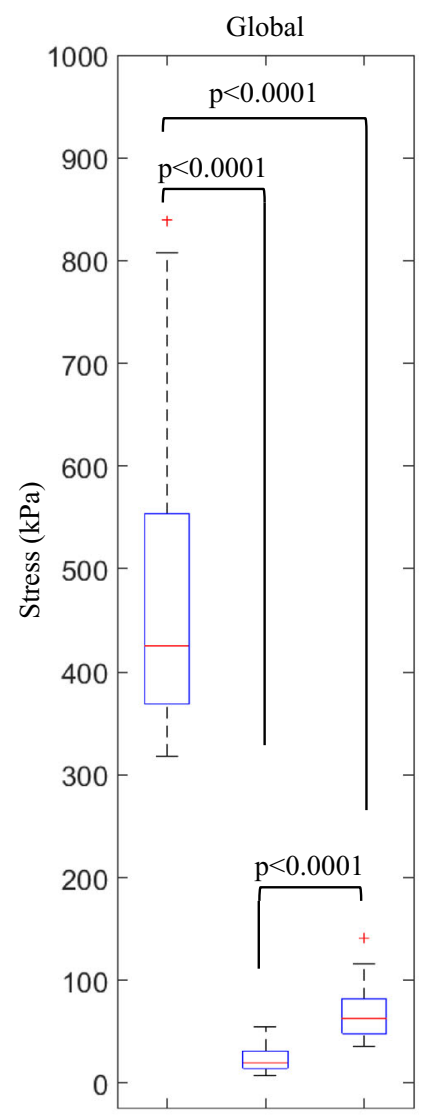

Axial Transverse Shear

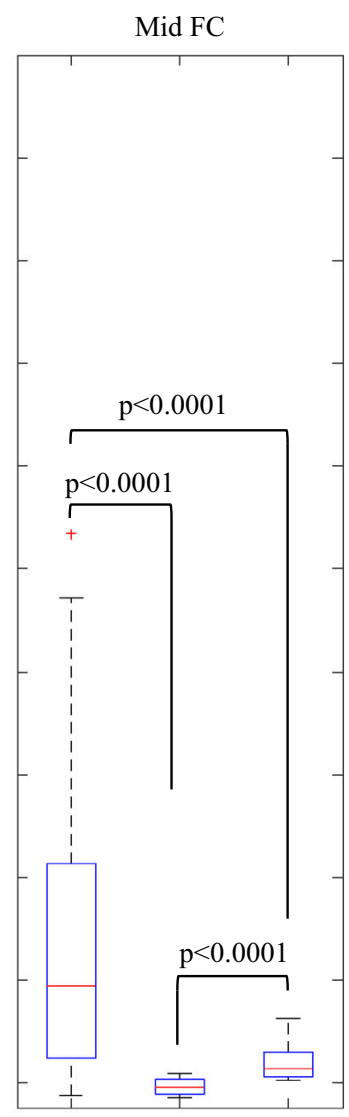

Axial Transverse Shear

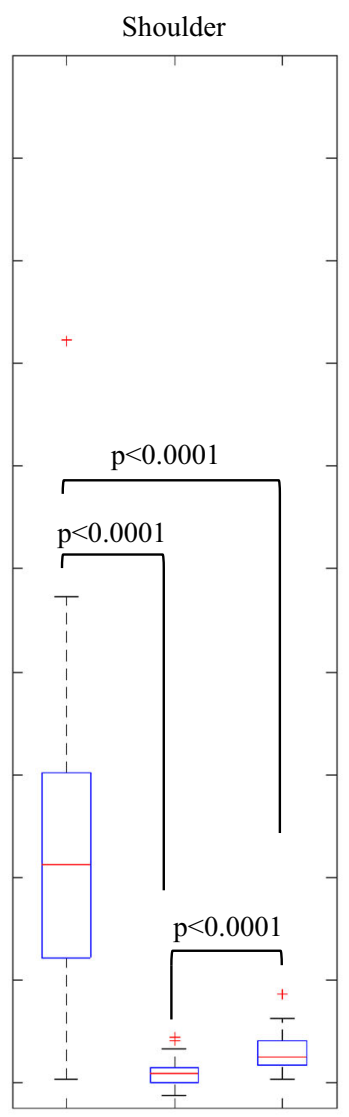

Axial Transverse Shear

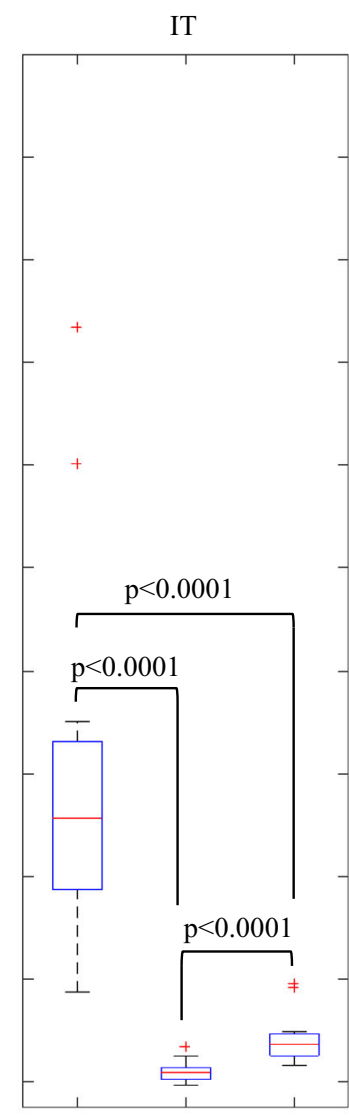

Axial Transverse Shear

FIGURE 6. Box-plot of different stress components (axial, transverse and shear stresses) globally and in different plaque regions (The red line marked the median value; upper and bottom edges of the box showed the 1st and 3rd quartile; the whiskers were for 1.5 times the interquartile range, and points outside the whiskers were marked individually as crosses.).

TABLE 5. Regression models of geometry parameters against fiber structure by region.

\begin{tabular}{|c|c|c|c|c|c|c|c|}
\hline \multirow[b]{2}{*}{ Fiber parameter } & \multirow[b]{2}{*}{ Geometry parameter } & \multicolumn{3}{|c|}{ Mid FC } & \multicolumn{3}{|c|}{ Shoulder } \\
\hline & & Slope & $\mathrm{R}^{2}$ & $p$ value & Slope & $\mathrm{R}^{2}$ & $p$ value \\
\hline \multirow[t]{3}{*}{ Fiber orientation $\left({ }^{\circ}\right)$} & FC thickness & 18.5 & 0.423 & 0.006 & 31.0 & 0.185 & 0.014 \\
\hline & Plaque burden & - & - & - & 30.0 & 0.161 & 0.023 \\
\hline & Normalized shoulder curvature & - & - & - & 0.471 & 0.163 & 0.022 \\
\hline \multirow[t]{3}{*}{ Fiber dispersion } & FC thickness & - & - & - & -0.164 & 0.168 & 0.020 \\
\hline & Normalized shoulder curvature & - & - & - & 0.003 & 0.165 & 0.021 \\
\hline & Lipid fraction & -0.124 & 0.325 & 0.021 & - & - & - \\
\hline
\end{tabular}

extend deeper into the tissue and approach the border of lipid and fibrous tissue. Loads are passed along rather than adjacent to fibers. Considering the relatively weaker material ${ }^{23}$ at the border region induced by inflammation, ${ }^{30}$ this transmitted loading may damage local tissue ${ }^{22}$ and eventually lead to FC rupture from the interior to the surface. ${ }^{9,12}$ Alternatively, the bands may suggest a mechanism allowing fractures initiated at the lumen surface, where stresses are highest, to propagate along the bands to the interior. ${ }^{13}$
Elastin and collagen are the principal fibers represented in the plaque structure. Orientation of a fiber defines the direction in which the fiber is stiffest and strongest. Indeed, mechanical loading is mostly distributed along the fibers, with axial stress by far the highest anisotropic component and nearly as high as anisotropic Stress-P ${ }_{1}$ (Figs. 4 and 6). Most studies report a modulus of elastin in the range $0.3-1.2 \mathrm{MPa}$ with a failure strain of up to $200 \%{ }^{1}$ Collagen has modulus of $600 \pm 200 \mathrm{MPa}$, a failure strain of 
TABLE 6. Regression models of geometry parameters against finite element analysis results by region.

\begin{tabular}{|c|c|c|c|c|c|c|c|}
\hline \multirow[b]{2}{*}{ FE result } & \multirow[b]{2}{*}{ Geometry parameter } & \multicolumn{3}{|c|}{ Mid FC } & \multicolumn{3}{|c|}{ Shoulder } \\
\hline & & Slope & $\mathrm{R}^{2}$ & $p$ value & Slope & $\mathrm{R}^{2}$ & $p$ value \\
\hline \multirow[t]{3}{*}{ Isotropic Stress- $P_{1}$} & Normalized shoulder curvature & -12.1 & 0.420 & 0.007 & 14.7 & 0.328 & 0.001 \\
\hline & Shoulder curvature & -38.6 & 0.408 & 0.008 & 54.7 & 0.366 & $<0.001$ \\
\hline & Lipid arc & 0.597 & 0.472 & 0.003 & - & - & - \\
\hline \multirow[t]{3}{*}{ Anisotropic Stress- $P_{1}$} & Normalized shoulder curvature & -30.0 & 0.324 & 0.021 & - & - & - \\
\hline & Shoulder curvature & -100.8 & 0.350 & 0.016 & - & - & - \\
\hline & Lipid arc & 1.38 & 0.316 & 0.024 & & & \\
\hline \multirow[t]{3}{*}{ Axial Stress } & Normalized shoulder curvature & -29.7 & 0.318 & 0.023 & - & - & - \\
\hline & Shoulder curvature & -99.8 & 0.343 & 0.017 & - & - & - \\
\hline & Lipid arc & 1.38 & 0.315 & 0.024 & - & - & - \\
\hline \multirow[t]{3}{*}{ Transverse Stress } & Normalized shoulder curvature & -1.76 & 0.390 & 0.010 & 1.43 & 0.449 & $<0.001$ \\
\hline & Shoulder curvature & -5.93 & 0.423 & 0.006 & 5.10 & 0.459 & $<0.001$ \\
\hline & Lipid arc & 0.085 & 0.418 & 0.007 & - & - & - \\
\hline \multirow[t]{4}{*}{ Shear Stress } & Normalized shoulder curvature & -3.06 & 0.303 & 0.027 & 1.07 & 0.133 & 0.040 \\
\hline & Shoulder curvature & -11.3 & 0.396 & 0.009 & - & - & - \\
\hline & Lumen area & 7.29 & 0.291 & 0.031 & - & - & - \\
\hline & Lipid area & 88.0 & 0.279 & 0.035 & - & - & - \\
\hline
\end{tabular}

TABLE 7. Regression models of isotropic Stress- $P_{1}$ against anisotropic Stress- $P_{1}$ and components of anisotropic stress, by region.

\begin{tabular}{|c|c|c|c|c|c|c|c|c|c|}
\hline \multirow[b]{2}{*}{ FE Result } & \multicolumn{3}{|c|}{ Mid FC } & \multicolumn{3}{|c|}{ Shoulder } & \multicolumn{3}{|c|}{ IT } \\
\hline & Slope & $\mathrm{R}^{2}$ & $p$ value & Slope & $\mathrm{R}^{2}$ & $p$ value & Slope & $\mathrm{R}^{2}$ & $p$ value \\
\hline Anisotropic Stress- $P_{1}$ & 2.364 & 0.703 & $<0.001$ & 0.509 & 0.316 & 0.001 & 1.174 & 0.712 & $<0.001$ \\
\hline Axial stress & 2.353 & 0.695 & $<0.001$ & 0.492 & 0.301 & 0.001 & 1.168 & 0.709 & $<0.001$ \\
\hline Transverse stress & 0.124 & 0.670 & $<0.001$ & 0.059 & 0.509 & $<0.001$ & - & - & - \\
\hline Shear stress & 0.196 & 0.432 & 0.006 & 0.078 & 0.462 & $<0.001$ & - & - & - \\
\hline
\end{tabular}

$13 \pm 2 \%$, and a failure stress of $60 \pm 10 \mathrm{MPa} .^{51}$ Considering the material strength and extensibility of the fibers, fracture of either elastin or collagen fibers is not expected under physiological conditions. The material structure between fiber bundles is weak relative to the fibers themselves ${ }^{28}$ and therefore has lower material strength. ${ }^{35}$ Anisotropic analysis revealed that transverse stress (stress pulling fibers apart or compressing fibers; Fig. 4d) was usually small (Fig. 6) and negative and likely contributes less to FC rupture. However, the pathological impact of compression in atherosclerosis remains unclear. Compared with transverse stress, shear stress was higher in the FC (Figs. 4e and 6), which may cause tissue sliding and tearing leading to structure failure. Experiments and clinical reports of failure modes plaque rupture are sparse and lack common terminology, but at least some suggest a role of delamination between fiber layers. ${ }^{13}$ This suggests fracture is the result of failure of the interfaces amongst fibers or between fibers and their surrounding matrix. Further, fiber damage commonly occurs in atherosclerotic plaques, including calcification of elastic fibers ${ }^{2}$ or cleavage of intact collagen fibers by matrix metalloproteinases. ${ }^{24}$ Fiber material strength and extensibility may decrease from these damages, permitting plaque rupture under physiological conditions due to the high stress along the fiber. More studies, in particular experimental studies, are needed to explore the relationship between the fiber alignment, pathological fiber features, and structure failure.

Mechanical analysis of human coronary plaques has been used to explain the location of plaque rupture. ${ }^{11,25,47}$ Such analysis shows potential as a novel tool to analyze the behavior of coronary plaques and can improve the ability of plaque imaging to predict different clinical presentations. ${ }^{5,41}$ A reliable modeling strategy is therefore needed for accurate and reproducible stress calculations. Including fiber structure caused a statistically significant change in Stress- $\mathrm{P}_{1}$ across the mid FC and IT regions, and individual plaques had considerable changes in predicted Stress$\mathrm{P}_{1}$ when fiber orientation was included (Fig. 5). These data imply a need to depict the fiber structure in vivo for plaque-specific analysis, which would represent a significant challenge for current imaging techniques. 
An ex vivo preliminary study has shown the potential to characterize fiber orientation in the aorta with a 7 Tesla magnetic resonance system. ${ }^{14}$ Further development of emerging imaging techniques, such as polarization-sensitive optical coherence tomography, ${ }^{27}$ may enable the in vivo identification of fiber thickness and orientation in the future.

It has to be pointed out that high stress concentration does not necessary lead to plaque rupture and symptom. However, it has been demonstrated that high stress is associated with clinical symptoms ${ }^{31,41,53}$ and subsequent major adverse event ${ }^{5,32}$ in both coronary and carotid circulations. By comparing stress level obtained from computational analysis between ruptured and unruptured lesions, Cheng et al., suggested a rupture threshold of $300 \mathrm{kPa} .{ }^{11}$ In this study, 8 out of 16 isotropic model cases had stresses over $300 \mathrm{kPa}$ in the key regions (all 8 in shoulder regions). With the anisotropic model, 7 of 16 cases had a region exceeding $300 \mathrm{kPa}$ (6 shoulders and $2 \mathrm{mid} \mathrm{FC}$, where both a shoulder and the mid FC in one case exceeded $300 \mathrm{kPa}$ ). Histology analysis with ruptured lesions showed that $37 \%(25 / 67)$ ruptures occurred in the mid $\mathrm{FC}$ and $63 \%$ in the shoulder region. ${ }^{29}$ In this study, most high stress concentrations were found to be located in the shoulder region in both isotropic and anisotropic models. This might be because histological slices used for analysis in this study had thick FC (2 cases with mean FC thickness between 100 and $200 \mu \mathrm{m}$ and the rest had FC thicker than $200 \mu \mathrm{m}$ ). The location of stress concentration could switch from shoulder region to the mid $\mathrm{FC}$ when $\mathrm{FC}$ is thin (e.g., $<200 \mu \mathrm{m}$ ). ${ }^{52}$ Moreover, apart from stress level, the material strength of the local tissue is a dominating factor for rupture. It is mainly determined by pathological conditions in the tissue (e.g., inflammatory burden) and geometry (e.g., thickness). Ideally, the combination of stress level and local material strength should be used to evaluate the rupture risk. Using current technologies, the material strength cannot be obtained noninvasively, but may be assessed indirectly from tissue thickness and pathological features, characterised by in vivo imaging. ${ }^{40}$

Histological stain, including picrosirius red, H\&E, and Masson's trichrome, and other microscopy techniques, e.g., polarized microscopy, electron microscopy, and fluorescence microscopy multiphoton microscopy, can be used to visualize collagen fibers. ${ }^{16,33}$ In this retrospective study, histological sections were stained using H\&E and EVG. Image processing was performed on slices stained by EVG harnessing its good contrast between elastin (in black) and collagen (in red) (Figs. 1 and 2); the one stained with $\mathrm{H} \& \mathrm{E}$ acted as a reference.

There are several limitations to this study. Firstly, fibers in different plaque regions and between different patients may have different material properties, which were not considered in the current analysis. Secondly, finite element models were constructed based on autopsy slides which could be subject to tissue damage and geometrical distortion during histological processing, despite our best efforts to minimize these. Thirdly, the number of subjects was small (sixteen plaques were analyzed), owing to the difficulty in collecting donors. Fourthly, static loading was used for the analysis. Micro-damage induced by the variation of loading during a cardiac cycle might accumulate resulting in material fatigue. ${ }^{21}$ This however was not considered in this study. Fifthly, the residual stress was not considered. Finally, analysis was limited to a $2 \mathrm{D}$ plane and the effect from blood flow was not considered.

In conclusion, significant differences were found in fiber architecture along the luminal region of human coronary atherosclerotic plaques. Fibers in the shoulder regions were more dispersed and less aligned with the lumen than those in the mid FC and IT regions. Considerable levels of shear stress exist in the FC, which may cause delamination between fibers, ultimately leading to $\mathrm{FC}$ rupture.

\section{ELECTRONIC SUPPLEMENTARY MATERIAL}

The online version of this article (doi: 10.1007/s10439-017-1827-3) contains supplementary material, which is available to authorized users.

\section{ACKNOWLEDGMENTS}

This research is supported by HRUK (RG2638/14/ 16), NSERC (6799-427538-2012), the WD Armstrong Trust, and the NIHR Cambridge Biomedical Research Centre.

\section{DISCLOSURE}

The authors do not have any conflict of interest to declare.

\section{OPEN ACCESS}

This article is distributed under the terms of the Creative Commons Attribution 4.0 International License (http://creativecommons.org/licenses/by/4.0/), which permits unrestricted use, distribution, and reproduction in any medium, provided you give appropriate credit to the original author(s) and the source, provide a link to the Creative Commons license, and indicate if changes were made. 


\section{REFERENCES}

${ }^{1}$ Aaron, B. B., and J. M. Gosline. Optical properties of single elastin fibres indicate random protein conformation. Nature 287:865-867, 1980.

${ }^{2}$ Bobryshev, Y. V. Calcification of elastic fibers in human atherosclerotic plaque. Atherosclerosis. 180:293-303, 2005. ${ }^{3}$ Bourantas, C. V., H. M. Garcia-Garcia, V. Farooq, A. Maehara, K. Xu, P. Genereux, R. Diletti, T. Muramatsu, M. Fahy, G. Weisz, G. W. Stone, and P. W. Serruys. Clinical and angiographic characteristics of patients likely to have vulnerable plaques: analysis from the prospect study. JACC Cardiovasc. Imaging. 6:1263-1272, 2013.

${ }^{4}$ Brown, A. J., D. R. Obaid, C. Costopoulos, R. A. Parker, P. A. Calvert, Z. Teng, S. P. Hoole, N. E. West, M. Goddard, and M. R. Bennett. Direct comparison of virtual-histology intravascular ultrasound and optical coherence tomography imaging for identification of thin-cap fibroatheroma. Circ. Cardiovasc. Imaging 8:e003487, 2015. ${ }^{5}$ Brown, A. J., Z. Teng, P. A. Calvert, N. K. Rajani, O. Hennessy, N. Nerlekar, D. R. Obaid, C. Costopoulos, Y. Huang, S. P. Hoole, M. Goddard, N. E. West, J. H. Gillard, and M. R. Bennett. Plaque structural stress estimations improve prediction of future major adverse cardiovascular events after intracoronary imaging. Circ. Cardiovasc. Imaging. 9(6):e004172, 2016.

${ }^{6}$ Burke, A. P., A. Farb, G. T. Malcom, Y. H. Liang, J. Smialek, and R. Virmani. Coronary risk factors and plaque morphology in men with coronary disease who died suddenly. N. Engl. J. Med. 336:1276-1282, 1997.

${ }^{7}$ Calvert, P., D. Obaid, N. West, L. Shapiro, D. McNab, C. Densem, P. Schofield, D. Braganza, S. Clarke, D. O'Sullivan, K. Ray, and M. Bennett. Association between virtual histology intravascular ultrasound findings and adverse outcomes in patients with coronary artery disease: The viva (vh-ivus in vulnerable atherosclerosis) study. JACC Imaging. 4:894-901, 2011.

${ }^{8}$ Chai, C. K., A. C. Akyildiz, L. Speelman, F. J. Gijsen, C. W. Oomens, M. R. van Sambeek, A. van der Lugt, and F. P. Baaijens. Local anisotropic mechanical properties of human carotid atherosclerotic plaques - characterisation by micro-indentation and inverse finite element analysis. $J$. Mech. Behav. Biomed. Mater. 43:59-68, 2015.

${ }^{9}$ Chapman, I. Morphogenesis of occluding coronary artery thrombosis. Arch. Pathol. 80:256-261, 1965.

${ }^{10}$ Cheng, J. M., H. M. Garcia-Garcia, S. P. de Boer, I. Kardys, J. H. Heo, K. M. Akkerhuis, R. M. Oemrawsingh, R. T. van Domburg, J. Ligthart, K. T. Witberg, E. Regar, P. W. Serruys, R. J. van Geuns, and E. Boersma. In vivo detection of high-risk coronary plaques by radiofrequency intravascular ultrasound and cardiovascular outcome: Results of the atheroremo-ivus study. Eur. Heart J. 35:639-647, 2014.

${ }^{11}$ Cheng, G. C., H. M. Loree, R. D. Kamm, M. C. Fishbein, and R. T. Lee. Distribution of circumferential stress in ruptured and stable atherosclerotic lesions. A structural analysis with histopathological correlation. Circulation 87:1179-1187, 1993.

${ }^{12}$ Constantinides, P. Pathogenesis of cerebral artery thrombosis in man. Arch. Pathol. 83:422-428, 1967.

${ }^{13}$ Daemen, M. J., M. S. Ferguson, F. J. Gijsen, D. S. Hippe, M. E. Kooi, K. Demarco, A. C. van der Wal, C. Yuan, and T. S. Hatsukami. Carotid plaque fissure: an underestimated source of intraplaque hemorrhage. Atherosclerosis. 254:102-108, 2016.
${ }^{14}$ Flamini, V., C. Kerskens, C. Simms, and C. Lally. Fibre orientation of fresh and frozen porcine aorta determined non-invasively using diffusion tensor imaging. Med. Eng. Phys. 35:765-776, 2013.

${ }^{15}$ Gao, H., Q. Long, Das S. Kumar, J. Halls, M. Graves, J. H. Gillard, and Z. Y. Li. Study of carotid arterial plaque stress for symptomatic and asymptomatic patients. J. Biomech. 44:2551-2557, 2011.

${ }^{16}$ Gasser, T. C., S. Gallinetti, X. Xing, C. Forsell, J. Swedenborg, and J. Roy. Spatial orientation of collagen fibers in the abdominal aortic aneurysm's wall and its relation to wall mechanics. Acta Biomater. 8:3091-3103, 2012.

${ }^{17}$ Gasser, T. C., R. W. Ogden, and G. A. Holzapfel. Hyperelastic modelling of arterial layers with distributed collagen fibre orientations. J. R. Soc. Interface 3:15-35, 2006.

${ }^{18}$ Haralick, R. M., and L. G. Shapiro. Computer and robot vision. Boston: Addison-Wesley Pub. Co., 1992.

${ }^{19}$ Heiland, V. M., C. Forsell, J. Roy, U. Hedin, and T. C. Gasser. Identification of carotid plaque tissue properties using an experimental-numerical approach. J. Mech. Behav. Biomed. Mater. 27:226-238, 2013.

${ }^{20}$ Holzapfel, G. A., G. Sommer, and P. Regitnig. Anisotropic mechanical properties of tissue components in human atherosclerotic plaques. J. Biomech. Eng. 126:657-665, 2004.

${ }^{21}$ Huang, Y., Z. Teng, U. Sadat, J. He, M. J. Graves, and J. $\mathrm{H}$. Gillard. In vivo mri-based simulation of fatigue process: A possible trigger for human carotid atherosclerotic plaque rupture. Biomed Eng Online. 12:36, 2013.

${ }^{22}$ Lee, R. T., F. J. Schoen, H. M. Loree, M. W. Lark, and P. Libby. Circumferential stress and matrix metalloproteinase 1 in human coronary atherosclerosis. Implications for plaque rupture. Arterioscler. Thromb. Vasc. Biol. 16:1070$1073,1996$.

${ }^{23}$ Lendon, C. L., M. J. Davies, G. V. Born, and P. D. Richardson. Atherosclerotic plaque caps are locally weakened when macrophages density is increased. Atherosclerosis. 87:87-90, 1991 .

${ }^{24}$ Libby, P. Collagenases and cracks in the plaque. $J$ Clin Invest. 123:3201-3203, 2013.

${ }^{25}$ Loree, H. M., R. D. Kamm, R. G. Stringfellow, and R. T. Lee. Effects of fibrous cap thickness on peak circumferential stress in model atherosclerotic vessels. Circ. Res. 71:850-858, 1992.

${ }^{26}$ Mozaffarian, D., E. J. Benjamin, A. S. Go, D. K. Arnett, M. J. Blaha, M. Cushman, et al. American Heart Association Statistics C., Stroke Statistics S. Heart disease and stroke statistics-2015 update: A report from the american heart association. Circulation 131:e29-322, 2015.

${ }^{27}$ Nadkarni, S. K., M. C. Pierce, B. H. Park, J. F. de Boer, P. Whittaker, B. E. Bouma, J. E. Bressner, E. Halpern, S. L. Houser, and G. J. Tearney. Measurement of collagen and smooth muscle cell content in atherosclerotic plaques using polarization-sensitive optical coherence tomography. $J$. Am. Coll. Cardiol. 49:1474-1481, 2007.

${ }^{28}$ O’Connell, M. K., S. Murthy, S. Phan, C. Xu, J. Buchanan, R. Spilker, R. L. Dalman, C. K. Zarins, W. Denk, and C. A. Taylor. The three-dimensional micro- and nanostructure of the aortic medial lamellar unit measured using $3 \mathrm{~d}$ confocal and electron microscopy imaging. Matrix Biol. 27:171-181, 2008.

${ }^{29}$ Richardson, P. D., M. J. Davies, and G. V. Born. Influence of plaque configuration and stress distribution on fissuring 
of coronary atherosclerotic plaques. Lancet 2:941-944, 1989.

${ }^{30}$ Rudd, J. H., E. A. Warburton, T. D. Fryer, H. A. Jones, J. C. Clark, N. Antoun, P. Johnstrom, A. P. Davenport, P. J. Kirkpatrick, B. N. Arch, J. D. Pickard, and P. L. Weissberg. Imaging atherosclerotic plaque inflammation with [18f]-fluorodeoxyglucose positron emission tomography. Circulation 105:2708-2711, 2002.

${ }^{31}$ Sadat, U., Z. Teng, V. E. Young, M. J. Graves, M. E. Gaunt, and J. H. Gillard. High-resolution magnetic resonance imaging-based biomechanical stress analysis of carotid atheroma: a comparison of single transient ischaemic attack, recurrent transient ischaemic attacks, non-disabling stroke and asymptomatic patient groups. Eur. J. Vasc. Endovasc. Surg. 41:83-90, 2011.

${ }^{32}$ Sadat, U., Z. Teng, V. E. Young, S. R. Walsh, Z. Y. Li, M. J. Graves, K. Varty, and J. H. Gillard. Association between biomechanical structural stresses of atherosclerotic carotid plaques and subsequent ischaemic cerebrovascular events-a longitudinal in vivo magnetic resonance imaging-based finite element study. Eur. J. Vasc. Endovasc. Surg. 40:485491, 2010.

${ }^{33}$ Schriefl, A. J., A. J. Reinisch, S. Sankaran, D. M. Pierce, and G. A. Holzapfel. Quantitative assessment of collagen fibre orientations from two-dimensional images of soft biological tissues. J. R. Soc. Interface 9:3081-3093, 2012.

${ }^{34}$ Shekhonin, B. V., S. P. Domogatsky, V. R. Muzykantov, G. L. Idelson, and V. S. Rukosuev. Distribution of type i, iii, iv and $\mathrm{v}$ collagen in normal and atherosclerotic human arterial wall: Immunomorphological characteristics. Coll Relat Res. 5:355-368, 1985.

${ }^{35}$ Sommer, G., S. Sherifova, P. J. Oberwalder, O. E. Dapunt, P. A. Ursomanno, A. DeAnda, B. E. Griffith, and G. A. Holzapfel. Mechanical strength of aneurysmatic and dissected human thoracic aortas at different shear loading modes. J. Biomech. 49:2374-2382, 2016.

${ }^{36}$ Sotomi, Y., R. A. Shlofmitz, S. Nakatani, Y. Onuma, and P. W. Serruys. Impact of the orbital atherectomy system on a coronary calcified lesion: quantitative analysis by light attenuation in optical coherence tomography. EuroIntervention. 11:e1, 2015.

${ }^{37}$ Stone, G. W., A. Maehara, A. J. Lansky, B. de Bruyne, E. Cristea, G. S. Mintz, R. Mehran, J. McPherson, N. Farhat, S. P. Marso, H. Parise, B. Templin, R. White, Z. Zhang, and P. W. Serruys. A prospective natural-history study of coronary atherosclerosis. N. Engl. J. Med. 364:226-235, 2011.

${ }^{38}$ Tang, D., Z. Teng, G. Canton, C. Yang, M. Ferguson, X. Huang, J. Zheng, P. K. Woodard, and C. Yuan. Sites of rupture in human atherosclerotic carotid plaques are associated with high structural stresses: an in vivo mribased 3d fluid-structure interaction study. Stroke 40:32583263, 2009.

${ }^{39}$ Tang, D., C. Yang, S. Kobayashi, J. Zheng, P. K. Woodard, Z. Teng, K. Billiar, R. Bach, and D. N. Ku. 3d mribased anisotropic fsi models with cyclic bending for human coronary atherosclerotic plaque mechanical analysis. $J$. Biomech. Eng. 131:061010, 2009.

${ }^{40}$ Tarkin, J. M., and J. H. Rudd. Techniques for noninvasive molecular imaging of atherosclerotic plaque. Nat Rev Cardiol. 12:79, 2015

${ }^{41}$ Teng, Z., A. J. Brown, P. A. Calvert, R. A. Parker, D. R. Obaid, Y. Huang, S. P. Hoole, N. E. West, J. H. Gillard, and M. R. Bennett. Coronary plaque structural stress is associated with plaque composition and subtype and higher in acute coronary syndrome: The beacon i (biomechanical evaluation of atheromatous coronary arteries) study. Circ. Cardiovasc. Imaging 7:461-470, 2014.

${ }^{42}$ Teng, Z., J. Feng, Y. Zhang, M. P. Sutcliffe, Y. Huang, A. J. Brown, Z. Jing, Q. Lu, and J. H. Gillard. A uni-extension study on the ultimate material strength and extreme extensibility of atherosclerotic tissue in human carotid plaques. J. Biomech. 48:3859-3867, 2015.

${ }^{43}$ Teng, Z., U. Sadat, G. Ji, C. Zhu, V. E. Young, M. J. Graves, and J. H. Gillard. Lumen irregularity dominates the relationship between mechanical stress condition, fibrous-cap thickness, and lumen curvature in carotid atherosclerotic plaque. J. Biomech. Eng. 133:034501, 2011.

${ }^{44}$ Teng, Z., U. Sadat, W. Wang, N. S. Bahaei, S. Chen, V. E. Young, M. J. Graves, and J. H. Gillard. Intraplaque stretch in carotid atherosclerotic plaque-an effective biomechanical predictor for subsequent cerebrovascular ischemic events. PLoS ONE 8:e61522, 2013.

${ }^{45}$ Teng, Z., Y. Zhang, Y. Huang, J. Feng, J. Yuan, Q. Lu, M. P. Sutcliffe, A. J. Brown, Z. Jing, and J. H. Gillard. Material properties of components in human carotid atherosclerotic plaques: a uniaxial extension study. Acta Biomater. 10:5055-5063, 2014.

${ }^{46}$ Thondapu V., Bourantas C. V., Foin N., Jang I. K., Serruys $\mathrm{P}$. W., Barlis P. Biomechanical stress in coronary atherosclerosis: emerging insights from computational modelling. Eur. Heart. J. 38:81-92, 2017.

${ }^{47}$ Vengrenyuk, Y., S. Carlier, S. Xanthos, L. Cardoso, P. Ganatos, R. Virmani, S. Einav, L. Gilchrist, and S. Weinbaum. A hypothesis for vulnerable plaque rupture due to stress-induced debonding around cellular microcalcifications in thin fibrous caps. Proc. Natl. Acad. Sci. USA 103:14678-14683, 2006.

${ }^{48}$ Virmani, R., F. D. Kolodgie, A. P. Burke, A. Farb, and S. M. Schwartz. Lessons from sudden coronary death : a comprehensive morphological classification scheme for atherosclerotic lesions. Arterioscler. Thromb. Vasc. Biol. 20:1262-1275, 2000.

${ }^{49}$ WHO. Global status report on noncommunicable diseases 2014. Geneva: World Health Organization, 2014.

${ }^{50} \mathrm{Xu}, \mathrm{C}$., J. M. Schmitt, S. G. Carlier, and R. Virmani. Characterization of atherosclerosis plaques by measuring both backscattering and attenuation coefficients in optical coherence tomography. J. Biomed. Opt. 13:034003, 2008.

${ }^{51}$ Yang, L., K. O. van der Werf, P. J. Dijkstra, J. Feijen, and M. L. Bennink. Micromechanical analysis of native and cross-linked collagen type i fibrils supports the existence of microfibrils. J. Mech. Behav. Biomed. Mater. 6:148-158, 2012.

${ }^{52}$ Yuan, J., Z. Teng, J. Feng, Y. Zhang, A. J. Brown, J. H. Gillard, Z. Jing, and Q. Lu. Influence of material property variability on the mechanical behaviour of carotid atherosclerotic plaques: a $3 \mathrm{~d}$ fluid-structure interaction analysis. Int J Numer Method Biomed Eng. 31(8):2015, 2015

${ }^{53}$ Zhu, C., Z. Teng, U. Sadat, V. E. Young, M. J. Graves, Z. Y. Li, and J. H. Gillard. Normalized wall index specific and mri-based stress analysis of atherosclerotic carotid plaques: A study comparing acutely symptomatic and asymptomatic patients. Circ. J. 74:2360-2364, 2010. 